\title{
Monotone type operators in nonreflexive Banach spaces
}

\author{
Yuqing Chen ${ }^{1}$ and Yeol Je Cho ${ }^{2,3 *}$
}

\author{
"Correspondence: yjcho@gnu.ac.kr \\ ${ }^{2}$ Department of Mathematics \\ Education and the RINS, Gyongsang \\ National University, Jinju, 660-701, \\ Korea \\ ${ }^{3}$ Department of Mathematics, \\ Faculty of Science, King Abdulaziz \\ University, Jeddah, 21589, Saudi \\ Arabia \\ Full list of author information is \\ available at the end of the article
}

\begin{abstract}
Let $E$ be a real Banach space, $E^{*}$ be the dual space of $E, E^{* *}$ be the dual space of $E^{*}$. Let $T: D(T) \subseteq E^{* *} \rightarrow 2^{E^{*}}$ be a monotone type mapping. In this paper, first, we introduce the special case when $T$ is the weak* sub-differential $\partial^{*} \phi$ of a convex function $\phi$ and obtain a surjective result for the mapping $\partial^{*}\left(\phi+\epsilon\|\cdot\|^{2}\right)$, where $\epsilon>0$. Second, we show the existence of solutions of the variational inequality problems for strictly quasi-monotone operators and semi-monotone operators. Finally, we construct a degree theory for mappings of the class $\left(S_{+}\right)$and then construct a generalized degree for the weak* sub-differential of a convex function.
\end{abstract}

\section{Introduction}

Monotone operators in reflexive Banach spaces has many applications in nonlinear partial differential equations, nonlinear semi-group theory, variational inequality and so on (see [1-4]). The theory for monotone operators in reflexive Banach spaces has been well developed. In recent years, many authors have generalized the monotone operator theory to nonreflexive Banach spaces. For example, maximal monotone operators in nonreflexive Banach spaces has been studied in [5-8] and variational inequality problems related to monotone type mappings in nonreflexive Banach spaces have been studied in [9-14]. For more references on variational inequality problems, see [15-24] and [25]. Also, degree theory for monotone type mappings in nonreflexive separable Banach spaces has been studied in [26, 27]. Also, see [3, 28-36] for more references on degree theory of monotone type operators.

In this paper, we study variational inequality problems and degree theory for monotone type mappings in nonreflexive spaces. This paper is organized as follows:

Let $E$ be a real Banach space, $E^{*}$ be the dual space of $E$ and $E^{* *}$ be the dual space of $E^{*}$. In Section 2, we introduce the weak" sub-differential $\partial^{*} \phi$ of a convex function $\phi: E^{* *} \rightarrow$ $R \cup\{+\infty\}$, which is a subset of the classical sub-differential, and we obtain $\partial^{*}\left(\phi+\epsilon\|\cdot\|^{2}\right)=$ $E^{*}$ for the sum of a lower semi-continuous convex function $\phi: E^{* *} \rightarrow R \cup\{+\infty\}$ in the weak* topology and $\epsilon\|x\|^{2}$, where $\epsilon>0$. In Section 3, we show the existence of solutions of variational inequality problems related to strictly quasi-monotone operators and semimonotone operators. In Section 4, we construct a degree theory for mappings of class $\left(S_{+}\right)$ and then construct a generalized degree for the weak" sub-differential of a convex function and obtain some degree results.

\section{基 Springer}

(92014 Chen and Cho; licensee Springer. This is an Open Access article distributed under the terms of the Creative Commons Attribution License (http://creativecommons.org/licenses/by/2.0), which permits unrestricted use, distribution, and reproduction in any medium, provided the original work is properly cited. 
Through this paper, we use $\rightarrow^{*}$ to represent the convergence in the weak* topology, $\rightarrow$ to represent the convergence in the weak topology and $\rightarrow$ represent the convergence in norm topology.

\section{The weak* sub-differential of convex functions}

In this section, let $E$ be a real Banach space, $E^{*}$ be the dual space of $E$ and $E^{* *}$ be the dual space of $E^{*}$.

Now, we introduce the weak* sub-differential of a convex function and study the solvability problems related this mapping.

First, we recall that the classical sub-differential of a convex function $\phi: E \rightarrow R \cup\{+\infty\}$ at $y$ is defined by

$$
\partial \phi(y)=\left\{f \in E^{*}: \phi(x)-\phi(y) \geq(f, x-y), \forall x \in D(\phi)\right\} .
$$

It is well known (Rockfellar [8]) that $\partial \phi$ is a maximal monotone mapping.

Definition 2.1 Let $\phi: E^{* *} \rightarrow R \cup\{+\infty\}$ be a convex function. Then

$$
\partial^{*} \phi(y)=\left\{f \in E^{*}: \phi(x)-\phi(y) \geq(f, x-y), \forall x \in D(\phi)\right\}
$$

is called the weak"sub-differential of $\phi$ at $y$.

It is obvious that $\partial^{*} \phi(y) \subseteq \partial \phi(y)$, but $\partial^{*} \phi(y)=\partial \phi(y)$ when $E$ is reflexive.

The following result is obvious.

Proposition 2.2 Let $\phi: E^{* *} \rightarrow R \cup\{+\infty\}$ be a convex function. Then we have the following:

(1) $\partial^{*} \phi(y)$ is a weak closed convex subset of $E^{*}$;

(2) $0 \in \partial^{*} \phi\left(y_{0}\right)$ if and only if $\phi\left(y_{0}\right)=\inf _{y \in D(\phi)} \phi(y)$;

(3) $\partial^{*} \phi: E^{* *} \rightarrow E^{*}$ is monotone.

Definition 2.3 (see [37]) Let $X$ be a topological space. A function $f: X \rightarrow R$ is said to be sequentially lower semi-continuous from above at $x_{0}$ if, for any sequence $\left\{x_{n}\right\}$ with $x_{n} \rightarrow x_{0}$, $f\left(x_{n+1}\right) \leq f\left(x_{n}\right)$ implies that $f\left(x_{0}\right) \leq \lim _{n \rightarrow \infty} f\left(x_{n}\right)$.

Similarly, $f$ is said to be sequentially upper semi-continuous from below at $x_{0}$ if, for any sequence $\left\{x_{n}\right\}$ with $x_{n} \rightarrow x_{0}, f\left(x_{n+1}\right) \geq f\left(x_{n}\right)$ implies that $f\left(x_{0}\right) \leq \lim _{n \rightarrow \infty} f\left(x_{0}\right)$.

Remark 1 It is well known that a lower semi-continuous function is a lower semicontinuous from above function, but the converse is not true and a lower semi-continuous from above and convex function with the coercive condition in a reflexive Banach space attains its minimum (see [37]). Also, it is well known that, for a convex function in a reflexive Banach space, lower semi-continuity in the strong topology is equivalent to lower semi-continuity in the weak topology, but this is not true for lower semi-continuity from above (see [38]). For more on lower semi-continuous from above functions with its generalizations and applications in nonconvex equilibrium problems, variational problems and fixed point problems, see [38-50] and [51]. 
Proposition 2.4 Let $\phi: E^{* *} \rightarrow R \cup\{+\infty\}$ be a convex function which is sequentially lower semi-continuous from above in the weak" topology and $\lim _{\|x\| \rightarrow+\infty} \phi(x)=+\infty$, then there exists $x_{0} \in E^{* *}$ such that $\phi\left(x_{0}\right)=\inf _{y \in D(\phi)} \phi(y)$.

Proof We take a sequence $\left\{x_{n}\right\}$ in $E^{* *}$ such that

$$
\phi\left(x_{1}\right) \geq \phi\left(x_{2}\right) \geq \cdots \geq \phi\left(x_{n}\right) \geq \cdots, \quad \phi\left(x_{n}\right) \rightarrow \inf _{x \in D(\phi)} \phi(x) .
$$

Since $\lim _{\|x\| \rightarrow+\infty} \phi(x)=+\infty$ and $\left\{x_{n}\right\}$ is a bounded sequence in $E^{* *}$, it follows that $\left\{x_{n}\right\}$ has a subsequence $\left\{x_{n_{k}}\right\}$ of $\left\{x_{n}\right\}$ with $x_{n_{k}} \rightarrow^{*} x_{0}$ in $E^{* *}$. By the assumption, since $\phi$ is sequentially lower semi-continuous from above, we have $\phi\left(x_{0}\right) \leq \lim _{n \rightarrow \infty} \phi\left(x_{n}\right)$ and so it follows that

$$
\phi\left(x_{0}\right)=\inf _{y \in D(\phi)} \phi(y) .
$$

This completes the proof.

Proposition 2.5 The function $\phi: E^{* *} \rightarrow R$ defined by $\phi(x)=\|x\|^{2}$ is sequentially lower semi-continuous in the weak" topology.

Proof Suppose $x_{n} \rightarrow^{*} x_{0}$. Then $x_{0}(f)=\lim _{n \rightarrow \infty} x_{n}(f)$ for all $f \in E^{*}$ and so

$$
\left|x_{0}(f)\right| \leq \liminf _{n \rightarrow \infty}\left\|x_{n}\right\|\|f\|
$$

for all $f \in E^{*}$. Thus we have

$$
\left\|x_{0}\right\|=\sup _{\|f\|=1}\left|x_{0}(f)\right| \leq \liminf _{n \rightarrow \infty}\left\|x_{n}\right\|
$$

and so $\left\|x_{0}\right\|^{2} \leq \liminf _{n \rightarrow \infty}\left\|x_{n}\right\|^{2}$. This completes the proof.

Theorem 2.6 Let $\phi: E^{* *} \rightarrow R \cup\{+\infty\}$ be a convex function which is sequentially lower semi-continuous in the weak"topology. Then we have

$$
\partial^{*}\left(\phi+\epsilon\|\cdot\|^{2}\right)\left(E^{* *}\right)=E^{*}
$$

for all $\epsilon>0$.

Proof For any $f \in E^{*}$, we set $\psi(x)=\phi(x)+\epsilon\|x\|^{2}-x(f)$ for all $x \in D(\phi)$. It is obvious that $\psi$ is sequentially lower semi-continuous in the weak" topology. Thus $\psi$ is sequentially lower semi-continuous from above in the weak* topology and

$$
\lim _{\|x\| \rightarrow+\infty} \psi(x)=+\infty
$$

By Proposition 2.4, there exists $x_{0} \in E^{* *}$ such that $\phi\left(x_{0}\right)=\inf _{x \in D(\psi)} \psi(x)$. By (2) of Proposition 2.2, $\left.0 \in \partial^{*}\left(\phi+\epsilon\|\cdot\|^{2}\right)-x(f)\right)\left(x_{0}\right)$, which is equivalent to $f \in \partial^{*}\left(\phi+\epsilon\|\cdot\|^{2}\right)\left(x_{0}\right)$. This completes the proof. 


\section{Existence of variational inequality problems}

In this section, we study variational inequality problems related to monotone type operators in nonreflexive Banach spaces.

First, we recall the following.

Definition 3.1 ([11]) A mapping $A(u, v): E^{* *} \times E^{* *} \rightarrow E^{*}$ is said to be semi-monotone if it satisfies the following conditions:

(1) for each $u \in E^{* *}, A(u, \cdot)$ is monotone, i.e., $(A(u, v)-A(u, w), v-w) \geq 0$ for all $v, w \in E^{* *}$;

(2) for each fixed $v \in E^{* *}, A(\cdot, v)$ is completely continuous, i.e., if $u_{j} \rightarrow u_{0}$ in weak ${ }^{*}$ topology of $E^{* *}$, then $A\left(u_{j}, v\right)$ has a subsequence $A\left(u_{j_{k}}, v\right)$ with $A\left(u_{j_{k}}, v\right) \rightarrow A\left(u_{0}, v\right)$ in norm topology of $E^{*}$.

Definition 3.2 ([15]) Let $E$ be a real Banach space and $T: D \subseteq E^{* *} \rightarrow 2^{E^{*}}$ be a mapping. $T$ is said to be strictly quasi-monotone if $(g, u-v)>0$ for all $u, v \in D$ and for some $g \in T v$ implies that $(f, u-v)>0$ for all $f \in T u$.

Remark 2 For quasi-monotone mappings, see [21].

Lemma 3.3 Let E be a real Banach space and $C$ be a nonempty bounded closed convex subset of $E^{* *}$. If $A: C \rightarrow 2^{E^{*}}$ is a finite dimensional weak* upper semi-continuous (i.e. for each finite dimensional subspace $F$ of $E^{* *}$ with $F \cap C \neq \emptyset, A: C \cap F \rightarrow 2^{E^{*}}$ is upper semi-continuous in the weak topology) and strictly quasi-monotone mapping with bounded closed convex values, then $\left(f_{v}, u_{0}-v\right) \leq 0$ for all $v \in C$ and for some $f_{v} \in T u_{0}$ if and only if $\left(g, u_{0}-v\right) \leq 0$ for all $v \in C$ and $g \in T v$.

Proof The proof is similar to Lemma 2.3 in [15], we omit the details.

Remark 3 For the results of Lemma 3.3 in monotone case, we refer to [10].

Theorem 3.4 Let $E$ be a real Banach space and $C$ be a nonempty weak* closed convex bounded subset of $E^{* *}$. If $A: C \rightarrow 2^{E^{*}}$ is a finite dimensional weakly upper semi-continuous and strictly quasi-monotone mapping with bounded closed convex values, then there exists $u_{0} \in C$ such that

$$
\left(f_{v}, u_{0}-v\right) \leq 0
$$

for all $v \in C$ and for some $f_{v} \in T u_{0}$.

Proof For any finite dimensional subspace $F$ of $E$ with $F \cap C \neq \emptyset$, let $j_{F}: F \rightarrow E$ be the natural inclusion and $j_{F}^{*}$ be the conjugate mapping of $j_{F}$. Consider the following variational inequality problem:

Find $u \in F \cap C$ such that

$$
\left(j_{F}^{*} f_{v}, u-v\right) \leq 0
$$

for all $v \in C \cap F$ and for some $f_{v} \in T u$. 
Since $T$ is finite dimensional weakly upper semi-continuous and $j_{F}^{*} T$ is upper semicontinuous on $F \cap C$, there exists $u_{F} \in F \cap C$ such that

$$
\left(j_{F}^{*} f_{v}, u_{F}-v\right) \leq 0
$$

for all $v \in C \cap F$ and for some $f_{v} \in T u_{F}$, i.e., $\left(f_{v}, u_{F}-v\right) \leq 0$ for all $v \in C \cap F$ and for some $f_{v} \in T u_{F}$. By Lemma 3.3, we get

$$
\left(g, u_{F}-v\right) \leq 0
$$

for all $v \in C \cap F$ and $g \in T v$. Now, we put

$$
W_{F}=\{u \in C:(g, u-v) \leq 0, \forall v \in F \cap C, g \in T v\} .
$$

It is obvious that $W_{F}$ is weak* closed convex. One can easily check that

$$
W_{\bigcup_{i=1}^{n} F_{i}} \subseteq W_{F_{i}}, \quad \operatorname{dim}\left(F_{i}\right)<+\infty, \quad F_{i} \cap C \neq \emptyset
$$

for $i=1,2, \ldots, n$. Hence $\bigcap_{F \in \mathcal{F}} W_{F} \neq \emptyset$, where

$$
\mathcal{F}=\{F \subset E: F \cap C \neq \emptyset, \operatorname{dim}(F)<+\infty\} .
$$

Take $u_{0} \in \bigcap_{F \in \mathcal{F}} W_{F}$. We claim that $u_{0}$ satisfies the conclusion of Theorem 3.4. In fact, $\left(g, u_{0}-v\right) \leq 0$ for all $v \in C$ and $g \in T v$. By Lemma 3.3, it follows that

$$
\left(f_{v}, u_{0}-v\right) \leq 0
$$

for all $v \in C$ and for some $f_{v} \in T u_{0}$. This completes the proof.

From Theorem 3.4, we have the following.

Corollary 3.5 Let $E$ be a real Banach space and $C$ be a nonempty weak" closed convex unbounded subset of $E^{* *}$. If $A: C \rightarrow 2^{E^{*}}$ is a finite dimensional weakly upper semi-continuous and strictly quasi-monotone mapping with bounded closed convex values and there exist $v_{0} \in C$ and $r>0$ such that

$$
\left(f, u-v_{0}\right)>0
$$

for all $f \in$ Tu and $u \in C$ with $\|u\|>r$, then there exists $u_{0} \in C$ such that

$$
\left(f_{v}, u_{0}-v\right) \leq 0
$$

for all $v \in C$ and for some $f_{v} \in T u_{0}$.

Proof If $C_{n}=C \cap B(0, n)$, then, by Theorem 3.4, there exists $u_{n} \in C_{n}$ such that

$$
\left(f, u_{n}-v\right) \leq 0
$$


for all $v \in C_{n}$ and for some $f_{v} \in T u_{n}$. By Lemma 3.3, we know that

$$
\left(g, u_{n}-v\right) \leq 0
$$

for all $v \in C_{n}$ and for some $g \in T v$. By the assumption, we know that $\left\|u_{n}\right\| \leq r$ for each $n=1,2, \ldots$ and thus we may assume that $u_{n} \rightarrow^{*} u_{0}$ as $n \rightarrow \infty$. Otherwise, we take a subsequence. Consequently, it follows that

$$
\left(g, u_{0}-v\right) \leq 0
$$

for all $v \in C$ and $g \in T v$. Again, if we use Lemma 3.3, we get the conclusion. This completes the proof.

Corollary 3.6 Let $E$ be a real Banach space, $B(0, R)=\left\{\|x\|<R: x \in X^{* *}\right\} \subset E^{* *}$ is the open ball centered at 0 with radius $R$. If $A: \overline{B(0, R)} \rightarrow E^{*}$ is a finite dimensional weakly continuous and strictly quasi-monotone mapping and

$$
(A u, u)>-\|A u\|\|u\|
$$

for all $u \in \partial B(0, R)$, then there exists $u_{0} \in B(0, r)$ such that $A u_{0}=0$.

Proof It is obvious that $\overline{B(0, R)}$ is weak" closed and convex. By Theorem 3.4, there exists $u_{0} \in \overline{B(0, R)}$ such that

$$
\left(A u_{0}, u_{0}-v\right) \leq 0
$$

for all $v \in B(0, R)$. Now, we claim that $A u_{0}=0$. First, we prove that $\left\|u_{0}\right\|<R$. In fact, if $\left\|u_{0}\right\|=R$, then, by the assumption, $\left\|A u_{0}\right\| \neq 0$ and thus there exists $v_{0} \in \partial B(0, R)$ such that $\left(A u_{0}, v_{0}\right)=-\left\|A u_{0}\right\|\left\|v_{0}\right\|$. But we have

$$
-\left\|A u_{0}\right\|\left\|u_{0}\right\|<\left(A u_{0}, u_{0}\right) \leq\left(A u_{0}, v_{0}\right)=-\left\|A u_{0}\right\|\left\|v_{0}\right\|,
$$

which is a contradiction. Therefore, we have $\left\|u_{0}\right\|<R$. Since there exists $r>0$ such that $u_{0}+v \in B(0, R)$ for all $v \in E^{* *}$ with $\|v\| \leq r$, we have

$$
\left(A u_{0}, v\right) \geq 0
$$

for all $v \in B(0, r)$ and so $A u_{0}=0$. This completes the proof.

Theorem 3.7 Let $K \subset E^{* *}$ be a bounded weak* closed convex subset. Suppose that $\phi$ : $E^{* *} \rightarrow R \cup\{+\infty\}$ is a lower semi-continuous convex function in the weak* topology $K \subseteq$ $D(\phi), A: K \times K \rightarrow E^{*}$ is semi-monotone, and $A(u, \cdot)$ is finite dimensional continuous for each $u \in K$. Then there exists $w_{0} \in K$ such that

$$
\left(A\left(w_{0}, w_{0}\right), u-w_{0}\right)+\phi(u)-\phi\left(w_{0}\right) \geq 0
$$

for all $u \in K$. 
Proof For each finite dimensional subspace $F$ of $E^{* *}$ with $F \cap K \neq \emptyset$, set $K_{F}=K \cap F$ and $\phi_{F}(x)=\phi(x)$ for $x \in F \cap D(\phi)$. By Theorem 2.5 in [11], there exists $u_{F} \in K_{F}$ such that

$$
\left(A\left(u_{F}, u_{F}\right), u-u_{F}\right)+\phi_{F}(u)-\phi_{F}\left(u_{F}\right) \geq 0
$$

for all $u \in K_{F}$. Let

$$
\mathcal{F}=\left\{F \subset E^{* *}: F \text { is finite dimensional subspace with } F \cap K \neq \emptyset\right\}
$$

and

$$
W_{F}=\{w \in K:(A(w, u), u-w)+\phi(u)-\phi(w) \geq 0\}
$$

By (3.1) and the monotonicity of $A\left(u_{F}, \cdot\right), W_{F}$ is a nonempty bounded subset. Denote by ${\overline{W_{F}}}^{*}$ the weak" closure of $W_{F}$. For any $F_{i} \in \mathcal{F}$ for each $i=1,2, \ldots, n$, it is easy to see that $W_{\bigcup_{i} F_{i}} \subset W_{F_{i}}$ for each $i=1,2, \ldots, n$. So, we have

$$
\bigcap_{F \in \mathcal{F}} \bar{W}_{F}^{*} \neq \emptyset .
$$

Let $w_{0} \in \bigcap_{F \in \mathcal{F}}{\overline{W_{F}}}^{*}$. Now, we prove that

$$
\left(A\left(w_{0}, w_{0}\right), u-w_{0}\right)+\phi(u)-\phi\left(w_{0}\right) \geq 0
$$

for all $u \in K$. For each $u \in K$, take $F \in \mathcal{F}$ such that $w_{0} \in K_{F}$ and $u \in K_{F}$. There exists $w_{j} \in W_{F}$ such that $w_{j} \rightarrow^{*} w_{0}$ and

$$
\left(A\left(w_{j}, u\right), u-w_{j}\right)+\phi(u)-\phi\left(w_{j}\right) \geq 0
$$

for each $j=1,2, \ldots$. By letting $j \rightarrow \infty$, the complete continuity of $A(\cdot, u)$ and weak" lower semi-continuity of $\phi$ imply that

$$
\left(A\left(w_{0}, u\right), u-w_{0}\right)+\phi(u)-\phi\left(w_{0}\right) \geq 0 .
$$

Set $u=t w_{0}+(1-t) v$ for all $t \in(0,1)$ and $v \in K$, by using the convexity of $\phi$ and letting $t \rightarrow 1$, we get

$$
\left(A\left(w_{0}, w_{0}\right), v-w_{0}\right)+\phi(v)-\phi\left(w_{0}\right) \geq 0
$$

This completes the proof.

\section{Degree theory for monotone type mapping}

In this section, assume that $E$ is always a real Banach space, $E^{*}$ is the dual space of $E$ and $E^{* *}$ is the dual space of $E^{*}$.

Definition 4.1 A set-valued operator $T: D(T) \subseteq E^{* *} \rightarrow 2^{E^{*}}$ is said to be strong to weak upper semi-continuous at $x_{0} \in D(T)$ if, for each weak open neighborhood $V$ of 0 in $E^{*}$ (i.e., open in the weak topology of $\left.E^{*}\right)$, there exists an open neighborhood $W$ of 0 in $E^{* *}$ such that $T y \cap\left(T x_{0}+V\right) \neq \emptyset$ for all $y \in x_{0}+W$. 
Definition 4.2 A set-valued operator $T: D(T) \subseteq E^{* *} \rightarrow 2^{E^{*}}$ is said to be a mapping of class $\left(S_{+}\right)$if the following conditions are satisfied:

(1) for each $x \in D(T), T x$ is a bounded closed convex subset;

(2) $T$ is strong to weak upper semi-continuous;

(3) if $x_{n} \in D(T), f_{n} \in T x_{n}$ for each $n \geq 1$ and $x_{j} \rightarrow^{*} x_{0}$ such that

$$
\varlimsup_{n \rightarrow \infty}\left(f_{n}, x_{n}-x_{0}\right) \leq 0,
$$

then $x_{n} \rightarrow x_{0} \in D(T)$ and $\left\{f_{n}\right\}$ has a subsequence $\left\{f_{n_{k}}\right\}$ with $f_{n_{k}} \rightarrow f_{0} \in T x_{0}$.

Definition 4.3 A family of set-valued operators $T_{t}: D \subseteq E^{* *} \rightarrow 2^{E^{*}}$ for all $t \in[0,1]$ is said to be a homotopy of mappings of class $\left(S_{+}\right)$if the following conditions are satisfied:

(1) for each $t \in[0,1], x \in D, T_{t} x$ is a bounded closed convex subset;

(2) $T_{t} x:[0,1] \times D \rightarrow E^{*}$ is strong to weak upper semi-continuous;

(3) if $x_{n} \in D(T), t_{n} \in[0,1], f_{n} \in T_{t_{n}} x_{n}$ for each $n \geq 1, t_{n} \rightarrow t_{0}$ and $x_{j} \rightarrow^{*} x_{0}$ such that

$$
\varlimsup_{n \rightarrow \infty}\left(f_{n}, x_{n}-x_{0}\right) \leq 0,
$$

then $x_{n} \rightarrow x_{0} \in D$ and $\left\{f_{n}\right\}$ has a subsequence $\left\{f_{n_{k}}\right\}$ with $f_{n_{k}} \rightarrow f_{0} \in T_{t_{0}} x_{0}$.

Definition 4.4 Let $T: D(T) \subseteq E^{* *} \rightarrow 2^{E^{*}}$ be a mapping satisfying the conditions (1) and (2) in Definition 4.1. Let $\left\{x_{j}\right\} \subset D(T)$ with $x_{j} \rightarrow^{*} x_{0} \in D(T)$ and $f_{j} \in T x_{j}$ with $f_{j} \rightarrow f_{0}$. If $\lim \sup _{j \rightarrow \infty}\left(f_{j}, x_{j}-x_{0}\right) \leq 0$ implies that

$$
f_{0} \in T x_{0}, \quad\left(f_{0}, x_{0}\right)=\lim _{j \rightarrow \infty}\left(f_{j}, x_{j}\right)
$$

then $T$ is called a generalized pseudo-monotone mapping.

Proposition 4.5 Let $T: D(T) \subseteq E^{* *} \rightarrow 2^{E^{*}}$ be a mapping of class $\left(S_{+}\right)$and $S: E^{* *} \rightarrow E^{*}$ be a mapping with closed convex values. Then the following conclusions hold:

(1) if $S$ is an upper semi-continuous and compact mapping, then $T+S$ is a mapping of class $\left(S_{+}\right)$;

(2) if $S$ is a generalized pseudo-monotone mapping and weak compact, i.e., $S$ maps bounded subsets in $E^{* *}$ to weak compact subsets in $E^{*}$, then $T+S$ is a mapping of class $\left(S_{+}\right)$.

For any subspace $F$ of $E^{* *}$, let $J_{F}: F \rightarrow E^{* *}$ be the natural inclusion and $J_{F}^{*}: E^{* * *} \rightarrow F^{*}$ be the conjugate mapping of $j_{F}$. Note that, under the canonical injection mapping $J: E^{*} \rightarrow$ $E^{* * *}$, i.e., $J x(f)=f(x)$ for all $f \in E^{* *}$ and $x \in E^{*}, E^{*}$ can be injected as a subspace of $E^{* * *}$ and so, in the following, we always regard $E^{*}$ as a subspace of $E^{* * *}$.

First, we need the following result from [36] (also, see [3]).

Lemma 4.6 Let $F$ be a finite dimensional subspace, $\Omega \subset F$ be an open bounded subset and let $0 \in \Omega$. Let $T: \bar{\Omega} \rightarrow 2^{F^{*}}$ be an upper semi-continuous mapping with compact convex values, $F_{0}$ be a proper subspace of $F, \Omega_{F_{0}}=\Omega \cap F_{0} \neq \emptyset$ and $T_{F_{0}}=j_{F_{0}}^{*} T: \overline{\Omega_{F_{0}}} \rightarrow 2^{F_{0}^{*}}$ be the Galerkin approximation of $T$, where $j_{F_{0}}^{*}$ is the adjoint mapping of the natural inclusion $j_{F_{0}}$ : $F_{0} \rightarrow F$. If $d(T, \Omega, 0) \neq d\left(T_{F_{0}}, \Omega_{F_{0}}, 0\right)$, then there exist $x \in \partial \Omega$ and $f \in T x$ such that $(f, x) \leq 0$ 
and $(f, v)=0$ for all $v \in F_{0}$, where $d(\cdot, \cdot, \cdot)$ is the topological degree for upper semi-continuous mappings with compact convex values in finite dimensional spaces (see Ma [52]).

Remark See $[53,54]$ for more references on degree theory of multivalued mappings.

Lemma 4.7 Let $T: \bar{\Omega} \rightarrow 2^{E^{*}}$ be a bounded mapping of $\left(S_{+}\right)$and let $0 \notin T(\partial \Omega)$. Then there exists a finite dimensional subspace $F_{0}$ of $E^{* *}$ such that

$0 \notin T_{F}(\partial \Omega \cap F)$

for all finite dimensional subspace $F$ of $E^{* *}$ with $F_{0} \subseteq F$, where $T_{F}=j_{F}^{*} T$.

Under the condition of Lemma 4.7, we know that $\operatorname{deg}\left(T_{F}, \Omega \cap F, 0\right)$ is well defined for the whole finite dimensional subspace $F$ of $E^{* *}$ with $F_{0} \subseteq F$, where $F_{0}$ is the same as in Lemma 4.7.

Lemma 4.8 Under the condition of Lemma 4.7, there exists a finite dimensional subspace $F_{0}$ of $E^{* *}$ such that $\operatorname{deg}\left(T_{F}, \Omega \cap F, 0\right)$ does not depend on $F$.

Now, let $\Omega \subset E^{* *}$ be a nonempty open bounded subset and $T: \bar{\Omega} \rightarrow 2^{E^{*}}$ be a mapping of class $\left(S_{+}\right)$. Suppose that $0 \notin T(\partial \Omega)$. In view of Lemmas 4.6 and 4.8 , we may define the topological degree as follows:

$$
\operatorname{deg}(T, \Omega \cap D(T), 0)=\operatorname{deg}\left(T_{F}, \Omega \cap F, 0\right),
$$

where $F$ is a finite dimensional subspace of $E^{* *}$ such that $F_{0} \subset F$ and $F_{0}$ is the same as in Lemma 4.8 .

Theorem 4.9 If $\operatorname{deg}(T, \Omega, 0) \neq 0$, then $0 \in T x$ has a solution in $\Omega$.

Proof The proof can be seen from the following proof of Theorem 4.10.

Theorem 4.10 Let $\left\{T_{t}\right\}_{t \in[0,1]}$ be a homotopy of mappings of class $\left(S_{+}\right)$. If $0 \notin T_{t}(\partial \Omega)$ for all $t \in[0,1]$, then $\operatorname{deg}\left(T_{t}, \Omega, 0\right)$ does not depends on $t \in[0,1]$.

Proof First, we claim that there exist finite dimensional subspaces $F_{0}$ of $E^{* *}$ such that $0 \notin$ $j_{F}^{*} T_{t}(\partial \Omega \cap F)$ for all finite dimensional subspaces $F$ with $F_{0} \subset F$. Suppose that this is not true. For any finite dimensional subspaces $F$, we define a set $W_{F}$ as follows:

$$
\begin{aligned}
& W_{F}=\left\{(t, x) \in[0,1] \times \partial \Omega: \text { there exists } f \in T_{t} x\right. \\
&\text { such that }(f, x) \leq 0 \text { and }(f, v)=0, \forall v \in F\} .
\end{aligned}
$$

Then $W_{F}$ is nonempty. Let $\overline{W_{F}}$ be the closure of $W_{F}$ in $[0,1] \times E^{* *}$ with $E^{* *}$ endowed with weak" topology. Consider the following family of sets:

$$
\mathcal{F}=\left\{\overline{W_{F}}: F_{0} \subset F, \operatorname{dim}(F) \leq \infty\right\} .
$$


It is easy to show that $\bigcap_{F \in \mathcal{F}} \overline{W_{F}} \neq \emptyset$. Let $\left(t_{0}, x_{0}\right) \in \bigcap_{F \in \mathcal{F}} \overline{W_{F}}$. If, for each $v \in E^{* *}$, we take a finite dimensional subspace $F$ such that $v \in F$ and $x_{0} \in F$, then there exist $\left(t_{j}^{v}, x_{j}^{v}\right) \in W_{F}$ and $f_{j}^{v} \in T_{t_{j}^{v}} x_{j}^{v}$ such that

$$
\begin{aligned}
& t_{j}^{v} \rightarrow t_{0}, \quad x_{j}^{v}-x_{0}, \\
& \left(f_{j}^{v}, x_{j}^{v}\right) \leq 0, \quad\left(f_{j}^{v}, v\right)=0
\end{aligned}
$$

for each $j \geq 0$. Hence we have

$$
\limsup _{j \rightarrow \infty}\left(f_{j}^{v}, x_{j}^{v}-x_{0}\right) \leq 0
$$

But, since $\left\{T_{t}: t \in[0,1]\right\}$ is a homotopy of mappings of class $\left(S_{+}\right)$, it follows that $x_{j}^{v} \rightarrow$ $x_{0} \in \partial \Omega$ and $\left\{f_{j}^{v}\right\}$ has a subsequence $\left\{f_{j_{k}}^{v}\right\}$ that converges weakly to $f_{0}^{v} \in T_{t_{0}} x_{0}$. Therefore, we have $\left(f_{0}^{v}, v\right)=0$. By Mazur's separation theorem (see [55]), we get $0 \in T_{t_{0}} x_{0}$, which is a contradiction. The claim is completed. So, it follows that $\operatorname{deg}\left(T_{t, F}, \Omega_{F}, 0\right)$ is well defined for the whole finite dimensional subspace $F$ with $F_{0} \subset F$.

Next, we prove that there exist a finite dimensional subspace $F_{1}$ and $F_{0} \subset F_{1}$ such that $\operatorname{deg}\left(T_{t, F}, \Omega_{F}, 0\right)$ does not depend on $t \in[0,1]$ for all finite dimensional subspace $F$ of $E^{* *}$ with $F_{1} \subset F$.

Suppose that this is not true. For any finite dimensional subspace $F$ with $F_{0} \subset F$, we define

$$
\begin{aligned}
& W_{F}=\left\{(t, x) \in[0,1] \times \partial \Omega: \text { there exists } f \in T_{t} x\right. \\
&\text { such that }(f, x) \leq 0 \text { and }(f, v)=0, \forall v \in F\} .
\end{aligned}
$$

Then $W_{F}$ is nonempty by Lemma 4.6 . Let $\overline{W_{F}}$ be the closure of $W_{F}$ in $[0,1] \times E^{* *}$ with $E^{* *}$ endowed with the weak** topology. Consider again the following family of sets:

$$
\mathcal{F}=\left\{\overline{W_{F}}: F_{0} \subset F \text { with } \operatorname{dim}(F) \leq \infty\right\} .
$$

It is easy to show that $\bigcap_{F \in \mathcal{F}} \overline{W_{F}} \neq \emptyset$. Let $\left(t_{0}, x_{0}\right) \in \bigcap_{F \in \mathcal{F}} \overline{W_{F}}$. Then, for each $v \in E^{* *}$, we take a finite dimensional subspace $F$ such that $F_{0} \subset F, v \in F$ and $x_{0} \in F$. Then there exist $\left(t_{j}^{v}, x_{j}^{v}\right) \in W_{F}$ and $f_{j}^{v} \in T_{t_{j}^{v}} x_{j}^{v}$ such that

$$
\begin{aligned}
& t_{j}^{v} \rightarrow t_{0}, \quad x_{j}^{v} \rightarrow x_{0}, \\
& \left(f_{j}^{v}, x_{j}^{v}\right) \leq 0, \quad\left(f_{j}^{v}, v\right)=0
\end{aligned}
$$

for $j \geq 0$. Hence we have

$$
\lim _{j \rightarrow \infty}\left(f_{j}^{v}, x_{j}^{v}-x_{0}\right) \leq 0 .
$$

But, since $\left\{T_{t}: t \in[0,1]\right\}$ is a homotopy of mappings of class $\left(S_{+}\right)$, we have $x_{j}^{v} \rightarrow x_{0} \in \partial \Omega$ and $f_{j}^{v}$ has a subsequence $\left\{f_{j_{k}}^{v}\right\}$ which converges weakly to $f_{0}^{v} \in T_{t_{0}} x_{0}$. Therefore, we have $\left(f_{0}^{v}, v\right)=0$. Again, by Mazur's separation theorem, $0 \in T_{t_{0}} x_{0}$, which is a contradiction. This completes the proof. 
Theorem 4.11 Let $T: \bar{\Omega} \rightarrow 2^{E^{*}}$ be a mapping of class $\left(S_{+}\right)$, where $\Omega \subset E^{* *}$ is an open bounded subset. If $0 \in \Omega$ and $(f, x)>0$ for all $x \in \partial \Omega \cap D(T)$ and $f \in T x$, then

$$
\operatorname{deg}(T, \Omega, 0)=1
$$

Proof Assume that $F$ is a finite dimensional subspaces of $E^{* *}$. It is straightforward to check that

$$
\left(j_{F}^{*} f, x\right)>0
$$

for all $x \in \partial \Omega \cap F$ and $f \in T x$. Therefore, we have $\operatorname{deg}\left(T_{F}, \Omega_{F}, 0\right)=1$ and so, by (4.1),

$$
\operatorname{deg}(T, \Omega, 0)=1 .
$$

Theorem 4.12 Let $T: E^{* *} \rightarrow 2^{E^{*}}$ be a bounded mapping of class $\left(S_{+}\right)$. If

$$
\lim _{\|x\| \rightarrow \infty} \inf _{f \in T x} \frac{(f, x)}{\|x\|}=+\infty
$$

then $T E^{* *}=E^{*}$.

Proof For each $p \in E^{*}$, we set $T_{1} x=T x-p$ for all $x \in E^{* *}$. Then it is easy to see that $T_{1}$ is a mapping of class $\left(S_{+}\right)$. One can easily see that $(f, x)>0$ for all $x \in \partial B(0, R), f \in T_{1} x$ and sufficiently large $R$. Thus, by Theorem $4.11, \operatorname{deg}\left(T_{1}, B(0, R), 0\right)=1$ and so, by Theorem 4.9, $0 \in T_{1} x$ has a solution in $B(0, R)$, i.e., $p \in T x$ has a solution in $B(0, R)$. This completes the proof.

In the following, we assume that $E^{* *}$ is separable and so we take any sequence $\left\{F_{n}\right\}$ of finite dimensional subspaces of $E^{* *}$ such that

$$
F_{1} \subset F_{2} \subset \cdots \subset F_{n} \subset \cdots, \quad \overline{\bigcup_{n=1}^{\infty} F_{n}}=E^{* *} .
$$

Lemma 4.13 Let $\phi: D(\phi) \subseteq E^{* *} \rightarrow R \cup\{+\infty\}$ be a lower semi-continuous convex function in the weak* topology, $\Omega \subset E^{* *}$ be open bounded and let $x_{1} \in D(\phi)$. Suppose that $\phi\left(x_{1}\right)<$ $\phi(x)$ for all $x \in \partial \Omega \cap D(\phi)$. Then there exists a positive integer $N$ such that

$$
0 \notin \partial \phi_{n}\left(\partial \Omega \cap F_{n}^{\prime} \cap D\left(\partial \phi_{n}\right)\right),
$$

where $\phi_{n}: F_{n}^{\prime} \rightarrow R \cup\{+\infty\}$ is a mapping defined by $\phi_{n}(x)=\phi(x)$ for all $x \in F_{n}^{\prime}$ and $F_{n}^{\prime}=$ $\operatorname{span}\left(F_{n} \cup\left\{x_{1}\right\}\right)$ for all $n>N$.

Proof Suppose that the conclusion is not true. There exists $x_{n} \in D(\phi)$ such that $0 \in \partial \phi_{n}\left(x_{n}\right)$ and so we have $\phi(x)-\phi\left(x_{n}\right) \geq 0$ for all $x \in F_{n}^{\prime} \cap D(\phi)$, which contradicts $\phi\left(x_{1}\right)<\phi(x)$ for all $x \in \partial \Omega \cap D(\phi)$.

Under the assumption of Lemma 4.13, we know that there exists a positive integer $N$ such that

$$
0 \notin \partial \phi_{n}\left(\partial \Omega \cap F_{n}^{\prime} \cap D\left(\partial \phi_{n}\right)\right)
$$


for all $n>N$ and so, by [32], $\operatorname{deg}\left(\partial \phi_{n}, \Omega \cap F_{n}^{\prime}, 0\right)$ is well defined. Now, we define a generalized degree as follows:

$$
\begin{aligned}
\operatorname{Deg} & \left(\partial^{*} \phi, \Omega \cap D\left(\partial^{*} \phi\right), 0\right) \\
= & \left\{k: \text { there exists } F_{n}, n \geq 1,\right. \text { satisfying (4.2) } \\
& \text { such that } \left.\operatorname{deg}\left(\partial \phi_{n_{j}}, \Omega \cap F_{n_{j}}^{\prime}, 0\right) \rightarrow k\right\} .
\end{aligned}
$$

Remark For generalized degree theory, see [56].

Theorem 4.14 Let $\phi: D(\phi) \subseteq E^{* *} \rightarrow R \cup\{+\infty\}$ be a lower semi-continuous convex function in the weak ${ }^{*}$ topology. If $\lim _{\|x\| \rightarrow+\infty} \phi(x)=+\infty$, then

$$
\operatorname{Deg}\left(\partial^{*} \phi, B(0, r) \cap D\left(\partial^{*} \phi\right), 0\right)=\{1\}
$$

for sufficiently large $r$.

Proof By the assumption $\lim _{\|x\| \rightarrow+\infty} \phi(x)=+\infty$, it follows from Proposition 2.4 that there exists $x_{0} \in D(\phi)$ such that $\phi\left(x_{0}\right)=\inf _{x \in D(\phi)} \phi(x)$ if we take a large enough $r$ such that $\phi\left(x_{0}\right)<$ $\phi(x)$ for all $x \in D(\phi) \cap \partial B(0, r)$.

For any $F_{n}(n \geq 1)$ satisfying (4.2), we put $F_{n}^{\prime}=\operatorname{span}\left(F_{n} \cup\left\{x_{0}\right\}\right)$. We may easily see that

$$
\phi_{n}\left(x_{0}\right)=\inf _{x \in F_{n} \cap D(\phi)} \phi\left(x_{n}\right)
$$

and so we have

$$
(f, x) \geq 0
$$

for all $x \in \partial B(0, r) \cap F_{n}^{\prime} \cap D\left(\partial \phi_{n}\right)$. Thus we have

$$
\operatorname{deg}\left(\partial \phi_{n}, B(0, r) \cap F_{n}^{\prime} \cap D\left(\phi_{n}\right), 0\right)=1
$$

and, consequently, we have

$$
\operatorname{Deg}\left(\partial^{*} \phi, B(0, r) \cap D\left(\partial^{*} \phi\right), 0\right)=\{1\} .
$$

This completes the proof.

\section{Competing interests}

The authors declare that they have no competing interests.

Authors' contributions

All authors contributed equally to the writing of this paper. All authors read and approved the final manuscript.

\section{Author details}

${ }^{1}$ College of Applied Mathematics, Guangdong University of Technology, Guangzhou, Guangdong 510006, P.R. China.

${ }^{2}$ Department of Mathematics Education and the RINS, Gyongsang National University, Jinju, 660-701, Korea.

${ }^{3}$ Department of Mathematics, Faculty of Science, King Abdulaziz University, Jeddah, 21589, Saudi Arabia. 


\section{Acknowledgements}

The second author was supported by the Basic Science Research Program through the National Research Foundation of Korea funded by the Ministry of Education, Science and Technology (NRF-2013053358).

Received: 27 February 2014 Accepted: 30 April 2014 Published: 15 May 2014

\section{References}

1. Barbu, V: Nonlinear Semigroups and Differential Equations in Banach Spaces. Noordhoff, Leyden (1976)

2. Brezis, H: Operateurs Maximaux Monotones. North-Holland, Amsterdam (1973)

3. O'Regan, D, Cho, YJ, Chen, YQ: Topological Degree Theory and Applications. Chapman and Hall/CRC Press, Boca Raton (2006)

4. Pascali, D, Sburlan, S: Nonlinear Mappings of Monotone Type. Noordhoff, Leyden (1978)

5. Borwein, JM: Maximality of sums of two maximal monotone operators in general Banach space. Proc. Am. Math. Soc. 135, 3917-3924 (2007)

6. Fitzpatrick, SP, Phelps, RR: Some properties of maximal monotone operators on nonreflexive Banach spaces. Set-Valued Anal. 3, 51-69 (1995)

7. Gossez, JP: On the range of a coercive maximal monotone operator in a nonreflexive Banach space. Proc. Am. Math Soc. 35, 88-92 (1972)

8. Rockafellar, RT: On the maximal monotonicity of subdifferential mapping. Pac. J. Math. 33, 209-216 (1970)

9. Beldiman, M: Equilibrium problems with set-valued mappings in Banach spaces. Nonlinear Anal. 68, 3364-3371 (2008)

10. Chang, SS, Lee, BS, Chen, YQ: Variational inequalities for monotone operators in nonreflexive Banach spaces. Appl. Math. Lett. 8, 29-34 (1995)

11. Chen, YQ: On the semi-monotone operator theory and applications. J. Math. Anal. Appl. 231, 177-192 (1999)

12. Domokos, A, Kolumban, J: Variational inequalities with operator solutions. J. Glob. Optim. 23, 99-110 (2002)

13. Verma, RU: Variational inequalities involving strongly pseudomonotone hemicontinuous mappings in nonreflexive Banach spaces. Appl. Math. Lett. 11,41-43 (1998)

14. Watson, PJ: Variational inequalities in nonreflexive Banach spaces. Appl. Math. Lett. 10,45-48 (1997)

15. Chen, YQ, Cho, YJ: On strictly quasi-monotone operators and variational inequalities. J. Nonlinear Convex Anal. 8, 391-396 (2007)

16. Fang, YP, Huang, NJ: Variational-like inequalities with generalized monotone mappings in Banach spaces. J. Optim. Theory Appl. 118, 327-338 (2003)

17. Fang, Z: Vector variational inequalities with semi-monotone operators. J. Glob. Optim. 32, 633-642 (2005)

18. Fang, Z: A generalized vector variational inequality problem with a set-valued semi-monotone mapping. Nonlinear Anal. 69, 1824-1829 (2008)

19. Guo, JS, Yao, JC: Variational inequalities with nonmonotone operators. J. Optim. Theory Appl. 80, 63-74 (1994)

20. Kassay, G, Kolumban, J, Pales, Z: Factorization of Minty and Stampacchia variational inequality systems. Eur. J. Oper. Res. 143, 377-389 (2002)

21. Karamardian, S, Schaible, S: Seven kinds of monotone maps. J. Optim. Theory Appl. 66, 37-46 (1990)

22. Lin, LJ, Yang, MF, Ansari, QH, Kassay, G: Existence results for Stampacchia and Minty type implicit variational inequalities with multivalued maps. Nonlinear Anal. 61, 1-19 (2005)

23. Minty, GJ: On the generalization of a direct method of calculus of variations. Bull. Am. Math. Soc. 73, $315-321$ (1967)

24. Pascali, D: On variational inequalities involving mappings of type (S). In: Nonlinear Analysis and Variational Problems. Springer, Berlin (2010)

25. Plubtieng, S, Sombut, K: Existence results for system of variational inequality problems with semimonotone operators. J. Inequal. Appl. 2010, 251510 (2010)

26. Wang, FL, Chen, YQ, O'Regan, D: Degree theory for ( $S+$ ) mappings in non-reflexive Banach spaces. Appl. Math. Comput. 202, 229-232 (2008)

27. Wang, FL, Chen, YQ, O'Regan, D: Degree theory for monotone type mappings in non-reflexive Banach spaces. Appl. Math. Lett. 22, 276-279 (2009)

28. Adres, J, Gorniewicz, L: Note on topological degree for monotone type multivalued maps. Fixed Point Theory 7 , 191-199 (2006)

29. Browder, FE: Fixed point theory and nonlinear problems. Bull. Am. Math. Soc. 1, 1-39 (1983)

30. Browder, FE: Degree theory for nonlinear mappings. Proc. Symp. Pure Math. Soc. 45, 203-226 (1986)

31. Chen, YQ, Cho, YJ: Topological degree theory for multi-valued mappings of class $\left(S_{+}\right)_{L}$. Arch. Math. 84, 325-333 (2005)

32. Chen, YQ, O'Donal, D, Wang, FL, Agarwal, R: A note on the degree for maximal monotone mappings in finite dimensional spaces. Appl. Math. Lett. 22, 1766-1769 (2009)

33. Kartsatos, AG, Skrypnik, IV: Topological degree theories for densely defined mappings involving operators of type $\left(S_{+}\right)$. Adv. Differ. Equ. 4, 413-456 (1999)

34. Kartsatos, AG, Skrypnik, IV: The index of a critical point for densely defined operators of type $\left(S_{+}\right)_{L}$ in Banach spaces. Trans. Am. Math. Soc. 354, 1601-1630 (2001)

35. Kartsatos, AG, Skrypnik, IV: A new topological degree theory foe densely defined quasibounded $\left(\tilde{S}_{+}\right)$-perturbations of multivalued maxima monotone operators in reflexive Banach spaces. Abstr. Appl. Anal. 2005, 121-158 (2005)

36. Zhang, SS, Chen, YQ: Degree theory for multivalued (S) type mappings and fixed point theorems. Appl. Math. Mech. $11,441-454(1990)$

37. Chen, YQ, Cho, YJ, Yang, L: Note on the results with lower semi-continuity. Bull. Korean Math. Soc. 39, 535-541 (2002)

38. Aruffo, A, Bottaro, G: Generalizations of sequential lower semicontinuity. Boll. Uni. Mat. Ital. Serie 9 1, $293-318$ (2008)

39. Aruffo, $A B$, Bottaro, $G$ : Some variational results using generalizations of sequential lower semi-continuity. Fixed Point Theory Appl. 2010, 323487 (2010)

40. Bugajewskia, D, Kasprzak, P: Fixed point theorems for weakly F-contractive and strongly F-expansive mappings. J. Math. Anal. Appl. 359, 126-134 (2009)

41. Castellania, M, Pappalardob, M, Passacantandob, M: Existence results for nonconvex equilibrium problems. Optim. Methods Softw. 25, 49-58 (2010) 
42. Al-Homidan, S, Ansari, QH, Yao, JC: Some generalizations of Ekeland-type variational principle with applications to equilibrium problems and fixed point theory. Nonlinear Anal. 69, 126-139 (2008)

43. Khanh, PQ, Quy, DN: A generalized distance and enhanced Ekeland's variational principle for vector functions. Nonlinear Anal. 73, 2245-2259 (2010)

44. Khanh, PQ, Quy, DN: On generalized Ekeland's variational principle and equivalent formulations for set-valued mappings. J. Glob. Optim. 49, 381-396 (2011)

45. Khanh, PQ, Quy, DN: On Ekeland's variational principle for Pareto minima of set-valued mappings. J. Optim. Theory Appl. 153, 280-297 (2012)

46. Lin, LJ, Du, WS: Ekeland's variational principle, minimax theorems and existence of nonconvex equilibria in complete metric spaces. J. Math. Anal. Appl. 323, 360-370 (2006)

47. Lin, LJ, Du, WS: On maximal element theorems, variants of Ekeland's variational principle and their applications. Nonlinear Anal. 68, 1246-1262 (2008)

48. Qiu, JH: On Has version of set-valued Ekelands variational principle. Acta Math. Sin. 28, 717-726 (2012)

49. Qiu, JH, He, F: P-distances, $q$-distances and a generalized Ekeland's variational principle in uniform spaces. Acta Math. $\operatorname{Sin} .28,235-254(2012)$

50. Qiu, JH, He, F: A general vectorial Ekeland's variational principle with a p-distance. Acta Math. Sin. 29, 1655-1678 (2013)

51. Chen, YQ, Cho, YJ, Kim, JK, Lee, BS: Note on KKM maps and applications. Fixed Point Theory Appl. 2006, 53286 (2006)

52. Ma, TW: Topological degree for set-valued compact vector fields in locally convex spaces. Diss. Math. 92, 1-43 (1972)

53. Gel'man, BD, Obukhovskii, W: New results in the theory of multivalued mappings, II. Analysis and applications. J. Sov. Math. 25, 123-197 (1993)

54. Gorniewicz, L: Topological Fixed Point Theory of Multivalued Mappings, 2nd edn. Topological Fixed Point Theory and Its Applications, vol. 4. Springer, Berlin (2006)

55. Rudin, W: Functional Analysis. MacGraw-Hill, New York (1973)

56. Petryshyn, WV: Generalized Topological Degree and Semilinear Equations. Cambridge University Press, Cambridge (1995)

10.1186/1687-1812-2014-119

Cite this article as: Chen and Cho: Monotone type operators in nonreflexive Banach spaces. Fixed Point Theory and Applications 2014, 2014:119

\section{Submit your manuscript to a SpringerOpen ${ }^{\odot}$ journal and benefit from:}

- Convenient online submission

- Rigorous peer review

- Immediate publication on acceptance

- Open access: articles freely available online

- High visibility within the field

- Retaining the copyright to your article 